\title{
HOW APPLIED MATHEMATICS BECAME PURE
}

\author{
PENELOPE MADDY \\ University of California, Irvine
}

\begin{abstract}
This paper traces the evolution of thinking on how mathematics relates to the worldfrom the ancients, through the beginnings of mathematized science in Galileo and Newton, to the rise of pure mathematics in the nineteenth century. The goal is to better understand the role of mathematics in contemporary science.
\end{abstract}

My goal here is to explore the relationship between pure and applied mathematics and then, eventually, to draw a few morals for both. In particular, I hope to show that this relationship has not been static, that the historical rise of pure mathematics has coincided with a gradual shift in our understanding of how mathematics works in application to the world. In some circles today, it is held that historical developments of this sort simply represent changes in fashion, or in social arrangements, governments, power structures, or some such thing, but I resist the full force of this way of thinking, clinging to the old school notion that we have gradually learned more about the world over time, that our opinions on these matters have improved, and that seeing how we reached the point we now occupy may help us avoid falling back into old philosophies that are now no longer viable. In that spirit, it seems to me that once we focus on the general question of how mathematics relates to science, one observation is immediate: the march of the centuries has produced an amusing reversal of philosophical fortunes. Let me begin there.

In the beginning, that is, in Plato, mathematical knowledge was sharply distinguished from ordinary perceptual belief about the world. According to Plato's metaphysics, mathematics is the study of eternal and unchanging abstract Forms, ${ }^{1}$ while science is uncertain and changeable opinion about the world of mere becoming. Indeed, in Plato's lights, of the two, only mathematics deserves to be called 'knowledge' at all! Of course if sense perception cannot give us knowledge, if mathematics is not about perceivable things, then Plato owes us an account of how we ordinary humans achieve this wonderful insight into the properties of the abstract world of Forms. Plato's answer is that we do not actually acquire mathematical knowledge at all; rather, we recollect it from a time before birth, when our souls, unencumbered by physical bodies, were free to commune with the Forms, and not just the mathematical ones, either - also Truth, Beauty, Justice, the Good, and so on. ${ }^{2}$ Whatever appeal this position may have held for the ancient Greeks, it will not begin to satisfy a contemporary, scientifically minded philosopher. But in any case, Plato's view

Received: August 6, 2007; in revised form: November 24, 2007

1 In fact, for various reasons, Plato may posit a kind of intermediate abstract entity, called a 'mathematical', between the Forms and the physical world. For discussion, see Wedberg (1955).

2 See, e.g., Silverman (2003). 
on the relative standing of mathematics and science is unambiguous: mathematics is the highest form of knowledge; science is mere opinion.

Of course 'science' for the Greeks was not what we call 'science' today; between the ancients and ourselves looms the scientific revolution, the beginning of experimental natural science as we know it. Pioneers of the new science like Galileo took mathematics to be central to God's design of the universe, as in this famous passage from 1610:

[Nature] is written in that great book which ever lies before our eyes - I mean the universe - but we cannot understand it if we do not first learn the language and grasp the symbols in which it is written. The book is written in the mathematical language, and the symbols are triangles, circles, and other geometric figures, without whose help it is impossible to comprehend a single word of it; without which one wanders in vain through a dark labyrinth. (as quoted by Kline, 1972, pp. 328-329. See also Machamer 1998a, pp. 64-65 for a slightly different translation.)

In the case of free fall, for example, Galileo notes that many accounts of its causes have been proposed, but he rejects this inquiry:

Such fantasies, and others like them, would have to be examined and resolved, with little gain. (Galileo, 1638, p. 159)

His idea is that we should concentrate on finding and testing a purely mathematical description of motion, which he goes on to do:

It suffices our Author that we understand him to want us to investigate and demonstrate some attributes of a motion so accelerated ... whatever be the cause of its acceleration ... that in equal times, equal additions of speed are made. (Galileo 1638, p. 159)

The universe operates according to mathematical laws, which we can uncover by bringing mathematics to bear on our observations. ${ }^{3}$ Notice the dramatic shift here: mathematics is not placed above science; rather, the two have become one. In the words of the mathematical historian Morris Kline:

mathematics became the substance of scientific theories.... The upshot ... was a virtual fusion of mathematics ... and science. (Kline, 1972, pp. 394-395)

The great thinkers of that time - from Descartes and Galileo to Huygens and Newton - did mathematics as science and science as mathematics without any effort to separate the two.

3 It should be noted that this bold proposal, later adopted by Newton (see below), did not meet with universal approval. Cf. Kline (1972), pp. 333-334: "First reactions to this principle of Galileo are likely to be negative. Description of phenomena in terms of formulas hardly seems to be more than a first step. It would seem that the true function of science had really been grasped by the Aristotelians, namely, to explain why phenomena happened. Even Descartes [who reacted against the Aristotelians] protested Galileo's decision to seek descriptive formulas. He said, 'Everything that Galileo says about bodies falling in empty space is built without foundation: he ought first to have determined the nature of weight.' Further, said Descartes, Galileo should reflect on ultimate reasons." Looking forward to Newton and his successors, Kline holds that "Galileo's decision to aim for description was the deepest and most fruitful idea that anyone has had about scientific methodology." 
Appealing as this picture may be, it is not the way we tend to see things today. What with the various shocks dealt in the interim to our intuitive sense of how an orderly mathematical universe should behave, a starker empiricism has come to replace the rationalistic tendencies of an earlier, simpler time. So, for example, early in the 20th century, the logical positivists of the famous Vienna Circle held that the meaning of any scientific assertion could be reduced in some way or other to the conditions under which it would be empirically verified. This idea did admirable service in eliminating much apparently empty philosophical talk as meaningless - Carnap gleefully classifies Heidegger's claim that "the Nothing itself nothings" as a pseudo-statement ${ }^{4}$ - but it also threatened to do the same for mathematics, an outcome that certainly did not appeal to the self-described scientific philosophers of the circle! Their solution was to view mathematics as purely linguistic, as true by the conventions of language, as telling us nothing contentful about the world. ${ }^{5}$ At the same time, those like Gödel who continued to maintain that mathematics provides substantial information about properly mathematical objects were confronted with a variation of Plato's problem: if the cognitive machinery of human beings works as we think it does, how can we gain knowledge of nonspatiotemporal, acausal entities? ${ }^{6}$ In all this, experimental natural science is taken as the paradigm of well-grounded knowledge and mathematics is called into question when it does not clearly measure up.

This is the reversal of philosophical fortunes I alluded to a moment ago: for Plato, mathematics is perfect knowledge and science is mere opinion; for the pioneers of the scientific revolution, mathematics and science are one; and for contemporary philosophers, science is the best knowledge we have and the status of mathematics is problematic. The movement from the cross-over point - when science and mathematics were identified - to our current state coincides roughly with the rise of pure mathematics, with the separation of mathematics from its worldly roots. Perhaps we can better understand where we are now if we reconsider how mathematics came to be peeled away from natural science in this way. This is a complex story, of course, but I hope to draw out three of its individual strands, ranging from the more mathematical to the more scientific perspective. ${ }^{7}$

I think the first of these strands, primarily a mathematician's eye view, is fairly familiar. Kline describes the situation this way:

... Descartes, Newton, Euler, and many others believed mathematics to be the accurate description of real phenomena ... they regarded their work as the uncovering of the mathematical design of the universe. (Kline, 1972, p. 1028)

Over the course of the 19th century, this picture changed dramatically:

... gradually and unwittingly mathematicians began to introduce concepts that had little or no direct physical meaning. (Kline, 1972, p. 1029)

Citing the rise of negative numbers, complex numbers, $n$-dimensional spaces, and noncommutative algebras, he remarks that "mathematics was progressing beyond concepts suggested by experience," but that "mathematicians had yet to grasp that their subject

4 See Carnap (1932, p. 69).

5 See Carnap (1950).

6 See, e.g., Gödel (1964) and Benacerraf (1973).

7 I should admit that I am no historian myself. As will become obvious, I draw heavily on the work of various real scholars in what follows. 
... was no longer, if it ever had been, a reading of nature" (Kline, 1972, p. 1030). By midcentury, the tide had turned:

... after about 1850, the view that mathematics can introduce and deal with ... concepts and theories that do not have immediate physical interpretation ... gained acceptance. (Kline, 1972, p. 1031)

This movement continued with the study of, for example, abstract algebras, pathological functions, and transfinite numbers. The heady new view of mathematics that accompanied this change is perhaps best expressed by Cantor:

Mathematics is entirely free in its development ... The essence of mathematics lies in its freedom. (as quoted in Kline, 1972, p. 1031)

This sentiment appears in the thinking of many of the most innovative mathematicians of the late 19th century; today, it is standard orthodoxy. Mathematics progresses by its own lights, independent of ties to the physical world. Legitimate mathematical concepts and theories need to have no direct physical interpretation.

A careful, systematic analysis of these developments would explain how mathematicians gradually came to see themselves as free to investigate whatever concepts, structures, or theories seem of sufficient mathematical interest or importance. Detailed historical studies would illustrate how particular mathematical inquiries are motivated by particular mathematical goals and values. To take just one example, consider the development of the concept of an abstract group. Though we now recognize the role of substitution groups and their subgroups in Galois' work around 1830, Galois himself never isolated the concept; that was left to Cayley some 20 years later. The surprise is that Cayleys version passed unnoticed, as did Dedekind's a decade later still, simply because there were not enough examples of groups to make the notion useful. It was not until the 1870s, when many diverse examples of groups had been identified - in Galois theory, number theory, geometry, and the theory of differential equations - that the idea of an abstract group caught on and flourished. Only at that point did it begin to serve a clear mathematical purpose: it calls attention to similarities between a broad range of otherwise quite dissimilar structures; it provides an elaborate and detailed general theory that can be applied in different contexts; and it produces illuminating diagnoses of the features responsible for particular phenomena ("that $x$ has feature $y$ is not due to its idiosyncrasies $z$ or $v$ or $w$ but only to its group structure'). The historical record also shows how the definition of the group concept was gradually shaped by the mathematical purposes for which it was intended; for example, when infinite groups entered the picture, cancellation laws were replaced by the requirement of inverses in the definition. Incidentally, it was not until the 1920 s that group theory entered physics, where it is now a central theme. ${ }^{8}$

This, then, is the first strand to the story of how mathematics came to separate from natural science - the pursuit of various purely mathematical goals gradually led mathematicians to new studies not motivated by their immediate application to the world - but again, I think this is only part of the story. Also worth recalling is a second familiar thread, most apparent in the evolution of attitudes toward geometry. In the beginning, it seems that even Euclid found the parallel postulate less obvious than the rest of his fundamental assumptions; after him, generations of geometers attempted to prove it from the others.

8 For more discussion and references, see Maddy (2007, §IV.3). 
By 1800 , several mathematicians held that the parallel postulate cannot be proved, that alternative geometries are logically consistent, but nevertheless that Euclidean geometry is the true theory of actual space.

The pivotal figure in this story is Gauss, whose efforts to prove the parallel postulate eventually led him, in his words, "to doubt the truth of geometry itself" (see Kline, 1972, p. 872). We have probably all heard the sometimes-disputed tale of Gauss measuring the sum of the angles of a triangle formed by three mountain tops, intending to test Euclid, only to conclude that the disparity fell within the margins of experimental error. In any case, it is beyond dispute that Gauss considered alternative geometries to be candidates for application to the physical world; the full flowering of this idea came when Gauss set the foundations of geometry as a topic for the qualifying exam of his student, Riemann. Of course it was Riemannian geometry that the mathematician Grossman recommended when Einstein consulted him in 1912.

With the confirmation of general relativity some years later, Euclidean geometry could no longer be regarded as true of physical space, but mathematicians were reluctant to classify it as straightforwardly false. Instead, they distinguished physical space from abstract mathematical space, or rather, from a full range of different abstract mathematical spaces, and Euclidean geometry was seen as true in some and false in others among these. (Resnik, 1997, p. 130, calls this sort of move a 'Euclidean rescue'.) At that point, it became natural to regard mathematicians as providing a well-stocked warehouse of abstract structures from which the natural scientist is free to select whichever tool best suits his needs in representing the world. ${ }^{9}$

This, then, is the second strand in the story of how mathematics pulled away from science. This time applications are involved, as they were not in the first, purely mathematical strand, though the tale of Euclidean rescue is still one visible primarily from the mathematician's point of view: mathematical theories are protected from empirical falsification by positing a special realm of abstracta about which they remain true. The moral for the natural scientist, for the application of mathematics, is that we now have a wide variety of mathematical options and that it may take delicate empirical investigation to determine which works best for a given application. Nevertheless, the mathematics that is successfully applied might still be regarded as the native language of the Book of Nature, just as Galileo understood it so long ago.

The third and final strand I would like to touch on here, perhaps less familiar than the two rehearsed so far, originates directly from the point of view of the natural scientist. We have so far considered aspects of the historical rise of pure mathematics, but over roughly the same period, there was a profound shift in the common understanding of how applied mathematics relates to the world. To get a feel for this, let us return to the scientific revolution, to Galileo's heir, Sir Isaac Newton.

Like Galileo, Newton views the world as mathematical in design; following Galileo in method also, he sets out to give a mathematical description of gravitational force without concern for the mechanism that produces it. ${ }^{10}$ In a famous passage at the end of the Principia, he writes,

9 See Maddy (2007, §§IV.2.iii and IV.4) for further discussion and references.

10 Cf. Kline (1972, p. 334): "we should note how completely Galileo's program was accepted by giants such as Newton." 
I have explained the phenomena of the heavens and of our sea by the force of gravity, but I have not yet assigned a cause to gravity. ... I do not feign hypotheses. For whatever is not deduced from the phenomena must be called a hypothesis; and hypotheses, whether metaphysical or physical, or based on occult quantities, or mechanical, have no place in experimental philosophy. ... it is enough that gravity really exists and acts according to the laws that we have set forth. (Newton, 1687, p. 943)

This move was especially liberating given the context that Newtonian gravity was not a natural fit for the prevailing Cartesian picture of action by contact forces, not to mention that the Cartesian doctrine of vortices in the plenum was highly problematic in its own right. ${ }^{11}$ Newton champions the mathematical description of the motions we can straightforwardly observe - what he calls 'manifest qualities' - over hypothetical explanations of those motions in terms of hidden causes. ${ }^{12}$

Altogether, Newton paints a remarkable mathematical portrait of the universe: from the general laws of motion and the law of universal gravitation, he explains the motions of the planets, the action of the tides, the trajectories of comets, the shape of the earth, and more, ${ }^{13}$ and he does so while inventing the required mathematics. The only weakness in all these is his mathematical conservatism. Unlike Leibniz, ${ }^{14}$ who developed the rudiments of the calculus at roughly the same time, Newton did not think of his mathematical techniques as constituting a general theory or method: rather than devising all-purpose algorithms, he was content to solve one individual problem after another. ${ }^{15}$ Worse yet for the progress of British mathematics was Newton's insistence on purely synthetic geometric methods. Though he sometimes used Descartes' analytic techniques, especially early on, he came to regard them as merely heuristic; the justifications in the Principia are overwhelmingly geometric. Kline writes:

11 See, e.g., Slowik (2005) for more on Descartes' physics. Cf. Smith (2002, pp. 141-142): “As Newton well realized ... no hypothetical contact mechanism seems even imaginable to effect 'attractive' forces among particles of matter generally. The Scholium (Newton, 1687, pp. 588589) thus occurs at the point where adherents to the mechanical philosophy would start viewing Newton's reasoning as 'absurd' (to use the word Huygens chose privately). The Scholium attempts to carry the reader past this worry, but not by facing the demand for a contact mechanism head-on. Instead, Newton warns that he is employing mathematically formulated theory in physics in a new way, with forces treated abstractly, independently of mechanism."

12 Cf. Shapiro (2002, p. 228): "Newton believed that by formulating his theories phenomenologically, in terms of experimentally observed properties, or principles deduced from them, without any causal explanations (hypotheses) of those properties, he could develop a more certain science."

13 Cf. Newton (1687, p. 382): "the basic problem of philosophy [i.e., natural science] seems to be to discover the forces of nature from the phenomena of motions and then to demonstrate the other phenomena from these forces. ... we derive from celestial phenomena the gravitational forces by which bodies tend toward the sun and toward the individual planets. Then the motions of the plants, the comets, the moon, and the sea are deduced from these forces."

14 For these methodological contrasts, see, e.g., Cohen \& Smith (2002a, pp. 20-22), Hall (2002), and Kline (1972, pp. 378-380).

15 Cf. Truesdell (1981, p. 98): "Newton's Principia ... is a monument of human achievement; it deserves the admiration and esteem of everyone. Should an engineer study it with a view to using its contents to determine the motion of a capsule projected into space, he would be gravelled. Motions there are in abundance, but no general equations. Each motion furnishes a new problem and is treated by itself. Examples there are, but no algorism: towering concepts and a magnificent approach, certainly, but no method." 
Newton did not really believe that he had departed from Greek geometry. Though he used algebra and coordinate geometry, which were not to his taste, he thought his underlying methods were but natural extensions of pure geometry. (Kline, 1972, p. 384) ${ }^{16}$

For Newton, as for Galileo, the language of the universe was synthetic geometry, ${ }^{17}$ and British mathematicians loyally, if ill-advisedly, continued in Newton's footsteps. ${ }^{18}$

Leibniz labored under no such restrictions. As Kline describes him, he

Was a man of vision who thought in broad terms, like Descartes. He saw the long-term implications of the new ideas and did not hesitate to declare that a new science was coming to light. (Kline, 1972, p. 384)

Leibniz's algebraic symbolic approach proved far more flexible and effective than Newton's synthetic geometry, and his continental followers were the ones to expand and improve the methods of the calculus. The historians Cohen and Smith write:

It was left to individuals within the Leibnizian tradition to recast Principia into the symbolic calculus. What became clear in this process was the superiority of purely symbolic methods ... With this realization the fundamental step in problems of physics ceased being one of finding an adequate geometric representation of the quantities involved, and instead

16 Incidentally, Stein notes Newton's claim, in the preface to Principia, that the principles of geometry are "obtained from other fields" and that "geometry is founded on mechanical practice" (Newton, 1687, p. 382) and remarks that "I am not aware of any other mathematician or philosopher of the seventeenth century who expressed such a view ... Gauss appears to have been the first mathematician of stature (after Newton) to have come - and only after a struggle to hold seriously the view that the grounds of geometry are empirical" (Stein, 1990, pp. 30, 44).

17 Cf. Guicciardini (2002, p. 323): "According to the Galilean tradition the Book of Nature is written in geometric terms. Newton endorsed this tradition."

18 See, e.g., Kline (1972, pp. 380-381): Because of the priority dispute between Newton and Leibniz, "the English and Continental mathematicians ceased exchanging ideas. Because Newton's major work and first publication on the calculus, the Principia, used geometrical methods, the English continued to use mainly geometry for about a hundred years after his death. The Continentals took up Leibniz's analytical methods and extended and improved them. These proved to be far more effective; so not only did the English mathematicians fall behind, but mathematics was deprived of contributions that some of the ablest minds might have made." When the British finally began to import Continental analysis in the early 19th century, the principles of Leibniz's $d y$ notation were called 'd-ism', as opposed to 'dot-age' for the use of Newton's $\dot{y}$ (see Kline, 1972, p. 622). See also Smith \& Wise (1989, pp. 151-152): “The [British] reformers originally saw it as their mission to bring the most powerful techniques of mathematical analysis ... to the moribund centres of mathematical non-learning in Britain. Of greatest immediate importance was replacing the cumbersome system of dots in the Newtonian fluxional notation with the $d$ 's of Leibnizian differentials. Symbols nearly as political as they were mathematical, the $d$ 's represented youth and progress in the modern age. Babbage later claimed that in 1812, as the Memoirs of the Analytical Society neared publication, he had suggested a more apt title: "The Principle of pure D-ism in opposition to the Dot-age of the University". His pun on deism suggests the ideology of natural law that he espoused and how it could be embedded in the symbols of mathematics. Dr. Thomson, while no deist in the religious sense, agreed ... that the 'inferiority' of dot-age had been 'a principle cause of the small progress made in later times by British mathematicians'." 
became one of formulating appropriate differential equations. (Cohen \& Smith, 2002a, p. 22) $)^{19}$

This was the job of the 18th century carried out largely on the continent.

This time the key figure is Euler. What we know as Newton's laws of motion - including the famous $F=m a$-were actually formulated by Euler in the mid-1700s, but that is just the tip of the iceberg:

Euler's mathematical productivity is incredible. His major mathematical fields were the calculus, differential equations, analytic and differential geometry of curves and surfaces, the theory of numbers, series, and the calculus of variations. This mathematics he applied to the entire domain of physics. He created analytic mechanics (as opposed to the older geometrical mechanics) and the subject of rigid body mechanics. He calculated the perturbative effect of celestial bodies on the orbit of a planet and the paths of projectiles in resisting media.... He investigated the bending of beams and calculated the safety load of a column.... He was first to treat the vibrations of light analytically and to deduce the equation of motion taking into account the dependence on the elasticity and density of the ether ... The fundamental differential equations for the motion of an ideal fluid are his. (Kline, 1972, pp. 401-402) ${ }^{20}$

Euler's collected works now run to nearly 90 volumes. ${ }^{21}$

In fact, the 18th century was tremendously productive in all these areas; perhaps it is not surprising that along the way, the foundational difficulties experienced by Newton and Leibniz were only exacerbated as mathematical analysis expanded and deepened. The momentous shift away from Newton's geometric methods toward the symbolic techniques of Leibniz not only opened the way to bold new developments at breathtaking speed

19 Cf. Truesdell (1968, pp. 92-93): "Except for certain simple if important special problems, Newton gives no evidence of being able to set up differential equations of motion for mechanical systems.... As we shall see, a large part of the literature of mechanics for sixty years following the Principia searches various principles with a view to finding the equations of motion for the systems Netwon had studied and for other systems nowadays though of as governed by 'Newtonian' equations."

20 See also Kline (1972, pp. 402-403): "Euler did not open up new branches of mathematics. But no one was so prolific or could so cleverly handle mathematics; no one could muster and utilize the resources of algebra, geometry, and analysis to produce so many admirable results. Euler was superbly inventive in methodology and a skilled technician. One finds his name in all branches of mathematics: there are formulas of Euler, polynomials of Euler, Euler constants, Euler integrals, and Euler lines."

21 Several of these volumes include scholarly introductions by Truesdell (1968, p. 106) who writes that "Euler was the dominating theoretical physicist of the eighteenth century. His work is undervalued in the usual, vague historical works.... The great bulk of [his] publication is not the only impediment to a just historical estimate of what he did. He put most of mechanics into its modern form; from his books and papers, if indirectly, we have all learned the subject, and his way of doing things is so clear and natural as to seem obvious. In fact, it was he who made mechanics simple and easy, and for the straightforward it is unnecessary to give references. In return, the scientist of today who consults Euler's later writings will find them perfectly modern, while other works of that period require effort and some historical generosity to be appreciated." Much more could be said in Euler's praise, but let me just add this stunning fact: "of the entire corpus of research on mathematics, theoretical physics, and engineering mechanics published from 1726 to 1800," his writings "alone account for approximately one third" (Calinger, 1975, p. 211). 
but also served to relax the level of rigor downward from the high standard traditionally associated with the Greeks and their followers; Lacroix remarks that "Such subtleties as the Greeks worried about we no longer need." 22 Kline describes the situation this way:

Eighteenth-century thinking was certainly loose and intuitive. Any delicate questions of analysis, such as the convergence of series and integrals, the interchange of the order of differentiation and integration, the use of differentials of higher order, and questions of existence of integrals and solutions of differential equations, were all but ignored. That the mathematicians were able to proceed at all was due to the fact that the rules of operation were clear. Having formulated the physical problems mathematically, the virtuosos got to work, and new methodologies and conclusions emerged. ... How could the mathematicians have dared merely to apply rules and yet assert the reliability of their conclusions? (Kline, 1972, p. 617)

The answer lies in the virtual identification of mathematics and natural science inherited from Galileo and Newton:

Their technical skill was unsurpassed; it was guided, however, not by sharp mathematical thinking but by intuitive and physical insights. ... The physical meaning of the mathematics guided the mathematical steps and often supplied partial arguments to fill in nonmathematical steps. The reasoning was in essence no different from a proof of a theorem of geometry, wherein some facts entirely obvious in the figure are used even though no axiom or theorem supports them. Finally, the physical correctness of the conclusions gave assurance that the mathematics must be correct. (Kline, 1972, pp. 400, 617)

Notice that if the result is a method that we do not quite recognize as mathematical, it also is not what we normally think of as physical either.

For our purposes, though, these subtleties are less important than the clear line of influence tracing back to Galileo and Newton. Euler remarks,

The generality I here take on ... reveals to us the true laws of Nature in all their brilliance. (as quoted by Truesdell, 1981, p. 113)

Kline summarizes that for the 18th century, "mathematics was simply unearthing the mathematical design of the universe" (Kline, 1972, p. 619). Clifford Truesdell, the great practitioner and historian of rational mechanics, remarks that its statements

are called phenomenological, because they represent the immediate phenomena of experience, not attempting to explain them in terms of corpuscles or other inferred (or hypothesized) quantities. (Truesdell, 1960, p. 22)

Thus, we find both key elements from Galileo and Newton continuing here: the conviction that mathematical theories truly represent the underlying mathematical structure of the

22 This appears in the 1810 preface to his three-volume compendium of 18th-century differential and integral calculus. See Kline (1972, p. 618) for the reference. 
world and endorsement of these theories as describing phenomena directly without appeal to theoretical hidden causes.

This same world view carries forward into the 19th century as well, perhaps best exemplified by Fourier's groundbreaking work on the dynamics of heat. In the opening sentences of what has been called his great 'mathematical poem', ${ }^{23}$ first published in 1822, Fourier announces that

Primary causes are unknown to us; but are subject to simple and constant laws, which may be discovered by observation, the study of them being the object of natural philosophy.

Heat, like gravity, penetrates every substance of the universe, its rays occupy all of space. The object of our work is to set forth the mathematical laws which this element obeys. (Fourier, 1822, p. 1) ${ }^{24}$

In the following paragraph, he makes explicit his connection to Galileo and Newton. ${ }^{25}$ At the end, he concludes:

The chief results of our theory are the differential equations of the movement of heat in solid or liquid bodies, and the general equation which relates to the surface. The truth of these equations is not founded on any physical explanation of the effects of heat. In whatever manner we please to imagine the nature of this element ${ }^{26}$ we shall always arrive at the same equations, since the hypothesis which we form must represent the general and simple facts from which the mathematical laws are derived. (Fourier, 1822, p. 464)

Once again, we see the conviction that nature is mathematical and that its laws can be directly observed and hold true whatever mechanisms may underlie them.

As it happens, a lively controversy arose between Fourier and Poisson, whose rival theory of heat appeared in $1835 .^{27}$ Poisson's objections actually first appear in a 1808 paper of Laplace, written in response to an early version of Fourier's work, where they trace to this Laplacian credo:

I have wanted to establish that the phenomena of nature reduce in the final analysis to action ad distans from molecule to molecule, and that the consideration of these actions ought to serve as the basis of the mathematical theory of these phenomena. (as quoted by Smith \& Wise 1989, p. 160)

23 See Smith \& Wise (1989, p. 149) for references.

24 See also Fourier (1822, p. 7): "Profound study of nature is the most fertile source of mathematical discoveries.... it is ... a sure method of forming analysis itself."

25 The only other predecessor mentioned is Archimedes, whose name often appears in such contexts; e.g., Kline (1972, p. 401) refers to Euler as "the man [of the eighteenth century] who should be ranked with Archimedes, Newton and Gauss." Machamer (1998a) makes the case for Galileo's debt to Archimedes.

26 "Whether we regard it as a distinct material thing which passes from one part of space to another, or whether we make heat consist simply in the transfer of motion."

27 Here I follow Wise (1981, pp. 23-29) and Smith \& Wise (1989, pp. 155-162). 
Poisson took this approach to heat, positing a complex microstructure of molecules and caloric fluid, ${ }^{28}$ summing (integrating) over their interactions and eventually generating a differential equation that differs from Fourier's by an extra term. This he embraced as representing a novel prediction of the theory - that conductivity varies with absolute temperature - subject to experimental test.

Fourier also begins with molecules, but instead of devising a theory to test, he works directly from experiment in the first place: first the observation that a warmer body loses heat to a cooler body at a rate proportional to the temperature difference; second that radiant heat does not penetrate a thin foil. He concludes that

If two molecules of the same body are extremely near, and are at unequal temperatures, that which is the most heated communicates directly to the other during one instant a certain quantity of heat; which quantity is proportional to the extremely small difference of the temperatures. (Fourier, 1822, pp. 456-457)

and

The layers in contact are the only ones which communicate their heat directly ... There is no direct action except between material points extremely near. (Fourier, 1822, p. 460)

By a subtle shift of the term 'molecule' from a physical body to an infinitesimal volume, Fourier establishes his differential equation - which he regards as "rest[ing] on observations alone rather than on any hypothesis as to the true nature of radiated heat" (Wise, 1981, p. 25) - so for all his talk of 'molecules', Fourier in fact treats heat as a continuous flow. From Fourier's perspective, Poisson's novel prediction can be easily accommodated at a certain point in his derivation if the purported variability is in fact observed; until then, its presence in the equation is an inappropriate extension of theory beyond experimental fact.

Given the stark contrast between their methodologies, Laplace and Poisson's objections to Fourier are predictable. Most fundamentally, the charge is that Fourier is masking the underlying physics: the differential heat equation is not the literal truth but an approximation (first, a finite sum over the molecules involved is treated as an integral, then the integral equation is transformed into the familiar differential equation). ${ }^{29}$ Of course, for Fourier, the differential equation is fundamental, a direct representation of observed behavior. A similar disagreement concerns the transition between the object under consideration and its environment; the historian Norton Wise puts it this way:

Because temperature to Laplace was a density of caloric, it could never change abruptly. Fourier, however, treated the boundary as a surface of

28 Cf. Smith \& Wise (1989, p. 160): "an explicit model of the relation between ponderable molecules and caloric fluid in a solid, incorporating both the free caloric radiated from the molecules and responsible for temperature, and the bound or latent caloric involved in changes of phase. His model attributed to the radiating molecules the full complexity of observable objects, including radiation to finite distances, radiation rates between molecules proportional to finite temperature differences, and a correction factor depending on absolute temperature to account for possible nonlinearity."

29 Cf. Wise (1981, p. 28): "For Laplace and Poisson ... the differential equations were not the fundamental representation of the physics; they were to be established only as transformations of integral equations, where the integrals represented physical sums over effects of isolated sources." 
no thickness across which the temperature jumped between internal and external values. [For Laplace,] an acceptable analysis would require a boundary of some thickness within which temperature changed gradually to the external value. (Wise, 1981, p. 26)

Given the unsatisfactory state of caloric theory, Fourier's position may be seen as running parallel to Newton's rejection of Descartes' vortices.

The denouement of this long story springs from a momentous scientific development that gathered energy over the course of the 19th century and culminated in the opening decades of the 20th. For all the talk of 'corpuscles' or 'atoms' or 'molecules' throughout the time period we have been reviewing, there was no responsible view of their nature - tiny infinitely hard ball bearings $?^{30}$ mathematical points? ${ }^{31}$ infinitesimal volumes? ${ }^{32}$ and the hypothesis of underlying, invisible, discrete structure of matter richly deserved its Newtonian expulsion from experimental natural science. But this began to change with Dalton's experimental work in the first decade of the 19th century. It is often surprising to those of us with little background in the history of science that the discipline we now know as chemistry was such a relative latecomer. While Galileo was studying free fall and Newton was writing the Principia, chemistry was largely alchemy; ${ }^{33}$ the beginning of 'modern' chemistry is typically set around 1750, linked to the work of Lavoisier, ${ }^{34}$ and a viable atomic theory of chemical combination began only with Dalton, roughly contemporary with Fourier.

Dalton proposed that a sample of an element consists of many identical atoms of constant weight, that the atoms of different elements are of different weight, that these atoms remain unchanged through chemical reactions, and that a chemical compound is composed of many identical molecules, each of which is composed of atoms of its constituent elements. This simple hypothesis immediately explained the known laws of chemical combination and then swept through chemistry during the first half of the 19th century; by 1860, stable atomic weights had been measured and confirmed and "the atom [came] into general acceptance as the fundamental unit of chemistry" (Ihde, 1964, p. 257). Beginning in mid-century, atomic theory spread into physics with the kinetic theory of Maxwell and Boltzmann. By the end of the 1800s, the atomic theory was a well-developed scientific hypothesis with considerable empirical support. ${ }^{35}$

Meanwhile, the 19th century also saw the invention and development of classical thermodynamics by Carnot and Clausius; its familiar second law states that entropy never

30 See, e.g., Smith \& Wise (1989, pp. 155-156): "Newton justified his programme through the 'analogy of nature', arguing that whatever held true for all observable objects has also to hold for their unobservable parts ... Since all observable objects possessed the qualities of extension, hardness, impenetrability, mobility, inertia, and gravitation, so also did its parts; they were infinitely hard atoms of finite size that attracted one another with a force varying as the inverse square of the distance between their centres, like perfect planets or marbles." Of course such a hypothesis would not appear in Newton's official experimental science (see Shapiro, 2002, p. 228).

31 As in Laplace, and before him, Boschovich. See Smith \& Wise (1989, p. 156).

32 As we have seen in Fourier.

33 Boyle (1627-1691) was an exception (see Partington, 1957, pp. 66-77). Newton was not; the so-called 'other Newton' spent much time and energy on alchemy (see Cohen \& Smith, 2002a, pp. 23-29).

34 See Ihde (1964, chapter 3) and Partington (1957, chapter VII).

35 For further discussion and references, see Maddy (1997, pp. 135-142, and 2007, §IV.5). 
decreases in a closed system, for example, that spilt milk does not spontaneously return to the glass. The physical chemist Jean Perrin sees Carnot as heir to Galileo, describing his 'inductive' method this way:

Each of these principles [of thermodynamics] has been reached by noting analogies and generalising the results of experience, and our lines of reasoning and statements of results have related only to objects that can be observed and to experiments that can be performed.... in the doctrine ... there are no hypotheses. (Perrin, 1913, p. vii)

Another physical chemist of the period, Pierre Duhem, begins his account of the history with Newton, cites Fourier with approval, and concludes that late-19th-century physicists have been "led ... gradually back to the sound doctrines Newton had expressed so forcefully" (Duhem, 1906, p. 53). Here we find a true successor to the purely phenomenological methods we have been tracing; the late-19th-century descendants of the Galileo-NewtonEuler-Fourier line regarded thermodynamics as "the very epitome of a scientific method of analogy and classification ... the apex of inductively derived ... science" (Nye, 1972, p. 34$){ }^{36}$

Thus the familiar battle lines were drawn between those who explain phenomena by appeal to hidden structures and those who describe what they see in terms of differential equations - except that this time the hypothesis in question, atomic theory, is more highly developed and empirically successful than ever before. Those opposed to hypotheses raised the familiar objection that atomic theory goes beyond experience; indeed, it was argued that atoms are in principle inaccessible to empirical test. ${ }^{37}$ To this general offense, one more specific was added, namely, that it conflicts with the favored theory, with classical thermodynamics:

[atomic theory] robbed Carnot's Principle of its claim to rank as an absolute truth and reduced it to the mere expression of a very high probability. (Perrin, 1913, p. 86)

According to kinetic theory, the spilt milk might spontaneously reassemble in the glass, though this is highly unlikely.

The conflict between the atomists and their thermodynamical opponents was so acute that Einstein, in one of his remarkable series of papers in 1905, sets out

to find facts which would guarantee as much as possible the existence of atoms of definite finite size. (Einstein, 1949, p. 47)

He does so by rederiving kinetic theory (because he was "not acquainted with earlier investigations of Boltzmann and Gibbs" [Einstein, 1949, p. 47]) and predicting in mathematical detail the behavior of "bodies of microscopically-visible size suspended in a liquid" (Einstein, 1905, p. 1). For all his efforts, Einstein was skeptical that experiments of the required precision were possible, ${ }^{38}$ but Perrin, the brilliant experimentalist, was in fact

36 Cf. Einstein (1949, p. 33): "[Thermodynamics] is the only physical theory of universal content concerning which I am convinced that, within the framework of the applicability of its basic concepts, it will never be overthrown."

37 Cf. Perrin (1913, p. 15): "It appeared to them more dangerous than useful to employ a hypothesis deemed incapable of verification."

38 See Nye (1972, p. 135). 
able to "prepare spherules of measurable radius" (Perrin, 1913, p. 114) and to confirm Einstein's predictions in a series of experiments on Brownian motion carried out around 1910. Poincaré, along with other leading skeptics, was immediately converted, declaring to a 1912 conference that "the atom of the chemist is now a reality." 39 And this consensus has only grown stronger since.

The upshot of this transformation, for our purposes, is an equally profound change in the understanding of how mathematics relates to the world. Before his conversion, Poincaré mused on the consequences that would follow if the kinetic theory should turn out to be correct:

Physical law will then take an entirely new aspect; it will no longer be solely a differential equation. (as quoted in Nye, 1972, p. 38)

Much as Laplace and Poisson insisted - though decidedly not for the reasons they gave! - the differential equation for heat flow is actually an approximation, an idealization, and what Richard Feynman describes as "a smoothed-out imitation of a really much more complicated microscopic world" (Feynman et al., 1964, pp. 12-12). The same applies for all the wonderful episodes of applied mathematics developed by Euler and his successors, and indeed, contemporary applied mathematicians take great care to determine precisely when various idealizations and simplifications that underlie their central differential equations can be counted both beneficial and benign.

To take just one example, consider the case of fluid dynamics. D. J. Tritton, the author of one recent textbook, observes:

The equations concern physical and mechanical quantities, such as velocity, density, pressure, temperature, which will be supposed to vary continuously from point to point throughout the fluid. How do we define these quantities at a point? To do so we have to make what is known as the assumption of the applicability of continuum mechanics or the continuum hypothesis. We suppose that we can associate with any volume of liquid, no matter how small, those macroscopic properties that we associate with the fluid in bulk... Now we know that this assumption is not correct if we go right down to molecular scales. We have to consider why is it nonetheless plausible to formulate the equations on the basis of the continuum hypothesis. (Tritton, 1988, p. 48)

Suppose, for example, that our model ${ }^{40}$ assigns a temperature to every point in a 3-dimensional volume. In fact, we know that temperature is an average energy over a group of molecules; the 'temperature' of our fluid point will function properly only if it successfully stands in for a small volume. Too small a volume will contain only a few molecules

39 As quoted in Nye (1972, p. 157). The two well-known opponents of atomic theory who were not converted - Duhem and Mach - both died in 1916. Wilson (2006, pp. 356-369, 654-660) argues that both were led to their anti-atomism in part because of real difficulties in the foundations of classical mechanics. Duhem also had religious motivations: he held that religious revelation, not physical science, is the proper source of information about underlying metaphysics (see Duhem, 1906, appendix).

40 Philosophers of science use the term 'model' in many senses; see Emch \& Liu $(2002, \S 1.3)$ for a bewildering survey. I use the term simply for an abstract mathematical object, ultimately (we might as well say) for a set (whose existence is presumably provable from the axioms of set theory). The 'temperature' assigned here is just a real number. 
that come and go at random, so its average energy will be subject to large fluctuations; too large a volume will include areas of significantly different average energies. "The applicability of the continuum hypothesis depends on there being a significant plateau" between these two extremes:

One may regard [the intermediate volume] as being an infinitesimal distance so far as macroscopic effects are concerned, and formulate the equations (as differential equations implicitly involving the limit of small separations) ignoring the behavior on still smaller length scales. (Tritton, 1988, p. 50)

There is more to it than that, but this gives the flavor of the applied mathematician's task.

But, even if the vaunted differential equations of Euler's analytical mechanics and the observationally perfect laws of thermodynamics are now regarded as "smoothed-out imitation[s] of a really much more complicated microscopic world" (Feynman et al., 1964, pp. 12-12), perhaps there remains room for literal description where continuum mathematics seems more fundamental. Unfortunately, there is little comfort from this quarter: consider, for example, the difficulties with the self-energy of a point particle in classical electrodynamics ${ }^{41}$ or the uncertainties about the small-scale structure of relativistic spacetime. ${ }^{42}$ It seems that our best hope actually lies in the opposite direction - in kinetic theory or statistical mechanics - where the underlying microstructure is taken seriously and the phenomenological principles of earlier theories are in some sense recovered from it.

The first triumph of kinetic theory is the derivation of the ideal gas law. ${ }^{43}$ Of course, this proof begins with a 'gas' made up of point masses that do not interact with each other and engage only in perfectly elastic collisions with the walls of the container. This is clearly an abstract model, not a description of real gases, for which the ideal gas law holds only under special conditions (at low densities and high temperatures) and even then only as a good approximation. The model can be improved by replacing the point masses with tiny hard spheres of finite radius and allowing for forces acting between them; the result is the van der Waals equation. ${ }^{44}$ Still, a van der Waals gas remains an abstract model, if one somewhat closer in structure to a real gas than the ideal gas model. This process can be continued, ${ }^{45}$ but in the end, the behavior of the gas elements is governed by quantum mechanics. Unfortunately, despite the stunning success of quantum mechanics as a predictive device, we still have no firm grasp of what worldly features underlie its various mathematical constructs. ${ }^{46}$

41 See Maddy (1997, pp. 147-149) for discussion and references.

42 See Maddy (1997, pp. 150-151) for discussion and references.

43 For textbook treatment, see, e.g., McQuarrie \& Simon (1997, §16-1) or Engel \& Reid (2006, $\S 16.1)$.

44 For textbook treatment, see, e.g., McQuarrie \& Simon (1997, §16-2, 16-7) or Brown et al. $(2006, \S 10.9)$.

45 Cf. McQuarrie \& Simon (1997, p. 648): “There are more sophisticated equations of state (some containing more than 10 parameters!) that can reproduce the experimental data to a high degree of accuracy over a large range of pressure, density and temperature" or Engel \& Reid (2006, p. 9): "there are other more accurate equations of state that are valid over a wider range than the van der Waals equation. Such equations of state include up to 16 adjustable substance-specific parameters."

46 See Maddy (2007, §§III.4, III.6) for discussion and references. 
The promise of literal description is perhaps greater in elementary statistical mechanics, where the reasoning is almost purely combinatorial. Whatever the small discrete elements of an isolated ${ }^{47}$ mole of gas are actually like, they can be properly described as occupying the left or the right half of the box that contains them. ${ }^{48}$ Suppose I am interested in what portion of the Avogadro's number of molecules is located in the left half of the box. I can straightforwardly calculate how many divisions of the individual molecules into onehalf or the other - how many 'microstates' - are configurations with, say, one-third of the molecules in the left half of the box. I can also calculate how many microstates there are all together, and I can compute the ratio of the first number to the second. This is all literal description of the situation.

If I now assume that all the microstates are equally likely, this ratio I have computed is the probability that one-third of the molecules are now in the left half of the box. With a few more such calculations, I begin to realize that this is dramatically less likely than something closer to a 50/50 split. Assuming my probabilistic assumption is correct, this is still straightforwardly literal, a matter of combinatorial fact about any collection of this large but finite size. It is comparable in status to $2+2=4$ as a description of the total number of fruits that result when we collect 2 apples and 2 oranges on the table. ${ }^{49}$

So far so good. But fairly soon, I find myself wanting to compute the most likely state directly. To do this, I consider a function $f$ from the set of configuration to the finite numbers, where $f(x$ molecules in the left side of the box $)=$ the number of microstates that place $x$ molecules on the left side of the box. The most likely state is the one where $f$ reaches a peak, that is to say, where the derivative of $f$ is 0 . But applying the methods of the differential calculus to $f$ does not really make sense, because the domain of $f$ is finite. To make this work, I treat the domain of $f$ as a continuous variable - and now I have taken leave of literal representation.

Ludwig Boltzmann, who pioneered this line of thought, apparently had finitistic leanings; he cautions his readers against forgetting that the underlying basis for his mathematical treatment lies in finite collections:

The concepts of the integral and differential calculus, cut loose from any [finitary] atomic representation, are purely metaphysical. (quoted with references and discussion in Emch \& Liu, 2002, p. 239)

In practical terms, the move from discrete sets to continuous mathematics actually takes place even earlier in our simple reasoning. The trouble is that determining the number of microstates involves computing $N$ !, where $N$ is Avogadro's number (approximately $6 \times 10^{23}$ ); given that 100 ! is already $9.3 \times 10^{157}$, the $N$ ! here is clearly out of feasible computational range. The standard solution is to work with $\ln (N !)$ and to use what is called Stirling's approximation: $N \times \ln (N)-N$. This move is justified because $\ln (N$ !)

47 Perfect thermal isolation is itself an idealization, of course, but let me set this aside. We might imagine that a small error factor has been included - so we only assume the box to be very well insulated - and that the error is too small to affect the main conclusions.

48 Determinate particle locations give way to probability densities over such locations in ordinary quantum theory, and further mysteries arise in attempts to formulate a relativistic quantum mechanics of particles (see Malament, 1996, for discussion). Here I am assuming that whatever our ultimate understanding of the structure of our mole of gas in the small, it will somehow reproduce enough particle structure to underwrite this reasoning from elementary statistical mechanics.

49 See Maddy (2007, §IV.2.ii) for more on the status of elementary arithmetic. 
is the sum of the $\ln (n)$ 's as $n$ varies from 1 to $N$, and if $N$ is large, this is very close to $\int_{1}^{N} \ln (x) \mathrm{d} x$, which is equal to Stirling's approximation. ${ }^{50}$ So computational practicality counsels the move from large finite to continuous even before the need for derivatives. I should note that this phenomenon - finite collections that are treated more successfully with infinitary, indeed continuum mathematics - is not at all special to statistical mechanics: in garden-variety statistics, discrete phenomena like household incomes or numbers of correct responses are routinely treated as continuous variables for similar reasons. ${ }^{51}$

In any case, this style of reasoning in statistical mechanics leads to Boltzmann's definition of entropy, the statistical version of the second law of thermodynamics, the statistical explanation of why real-world processes seem irreversible when the underlying mechanics is reversible, ${ }^{52}$ and more practically, to the physical chemist's ability to predict the direction of chemical reactions. ${ }^{53}$ Efforts to justify the mathematical methods employed in these accounts involve departures from literal description of a more conceptual nature than those discussed so far: for example, in ergodic theory, one considers, among other things, the evolution of a system as time goes to infinity; in the theory of the thermodynamic limit, one takes a limit as the number of molecules in the system goes to infinity. ${ }^{54}$ So beyond its first baby steps, statistical mechanics also departs substantially from literal description.

Under the circumstances, I think it is fair to characterize the work of contemporary scientists as presenting mathematical models of physical systems much as Duhem describes them in this passage: ${ }^{55}$

When a physicist does an experiment, two very distinct representations of the instrument on which he is working fill his mind: one is the image of the concrete instrument that he manipulates in reality; the other is a schematic model of the same instrument, constructed with the aid of symbols supplied by theories; and it is on this ideal and symbolic instrument that he does his reasoning, and it is to it that he applies the laws and formulas of physics. (Duhem, 1906, pp. 155-156)

A manometer, for example, is

On the one hand, a series of glass tubes, solidly connected to one another ... filled with a very heavy metallic liquid called mercury by the

50 See, e.g., McQuarrie \& Simon (1997, pp. 809-815) or Arfken (1985, pp. 555-558) for textbook discussions. This method appears in introductory treatments, e.g., Halliday et al. (2005, pp. 552-553) or Engel \& Reid (2006, pp. 284-285).

51 For a textbook example, see Rice (2007, p. 60).

52 See, e.g., Emch \& Liu (2002, pp. 106-112).

53 For textbook treatments, see Brown et al. (2006, chapter 19) or McQuarrie \& Simon (1997, chapters 20-22).

54 See Uffink (2007, §§6.1 and 6.3, respectively).

55 There is some irony in the appeal to Duhem here, as he was one of the last anti-atomists. Obviously, his embrace of Newton's 'sound doctrines' did not include the idea that mathematics correctly describes reality; Duhem (1906, pp. 133-134) actually harkens back to Poisson's objections to Fourier, e.g., "the body studied is geometrically defined; its sides are true lines without thickness." In fact, Duhem is a fascinating case: he opposed those who formed hypotheses, but he also opposed those who took differential equations literally, perhaps for reasons of the sort noted in footnote 39. This drove him to an unappealing fictionalism about natural science (see below). 
chemists; on the other hand, a column of that creature of reason called a perfect fluid in mechanics, and having at each point a certain density and temperature defined by a certain equation of compressibility and expansion. (Duhem, 1906, pp. 156-157)

There would be little point to such talk if the relation between the model and the physical system were a straightforward isomorphism, but the story just recounted shows that this is not true for the ubiquitous differential equations of applied mathematics and (with minor exceptions ${ }^{56}$ ) we do not appear to be in a position to make any stronger claims about subsequent theories that use other types of mathematics. In fact, the exact structure of those relations varies from case to case, as does our level of understanding of them. When we represent a cannonball as a perfect sphere; the lengths, times, angles, and forces involved as real numbers; the local surface of the earth as flat; and so on, in order to determine where a given ball, fired with a given force, will land, we have a fairly good idea of at least some of our departures from literal truth and why they are admissible. When we represent spacetime as a continuous manifold, we are not entirely sure whether or not this constitutes a literal truth, though our well-informed hunch is that even if it is an idealization, it is a good one - much as Euclidean geometry is a good approximation to the truth in most ordinary cases. But the fact remains that mathematics has been peeled away from science; the actual claim the scientist makes about the world is that it is probably, at least approximately, similar in structure to the mathematical model in certain respects and that the idealizations involved are beneficial and benign for the purposes at hand.

We have now viewed the rise of pure mathematics from several vantage points. First, we have seen how the study of many pure mathematical concepts, structures, and theories arose simply because mathematicians began to pursue a range of peculiarly mathematical goals with no immediate connection to applications. Second, we have seen how Euclidean geometry, once unblushingly regarded as the true theory of physical space, became the study of one among many abstract mathematical spaces. Third and finally, we have seen how our best mathematical accounts of physical phenomena are not the literal truths Newton took them for but freestanding abstract models that resemble the world in ways that are complex and sometimes not fully understood. Paradoxical as it may sound, it now appears that even applied mathematics is pure.

This, then, is the story behind my title, 'How applied mathematics became pure'. This story has morals, it seems to me, about how mathematics functions both in application and in its pure pursuit. One clear moral for our understanding of mathematics in application is that we are not in fact uncovering the underlying mathematical structures realized in the world; rather, we are constructing abstract mathematical models and trying our best to make true assertions about the ways in which they do and do not correspond to the physical facts. There are rare cases where this correspondence is something like isomorphism - we have touched on elementary arithmetic and the simple combinatorics of beginning statistical mechanics, and there are probably others, like the use of finite group theory to describe simple symmetries - but most of the time, the correspondence is something more complex, and all too often, it is something we simply do not yet understand: we do not know the small-scale structure of space-time or the physical structures that underlie quantum mechanics. And even this leaves out the additional approximations and

56 That is, for the likes of ' $2+2=4$ ' and elementary statistical mechanics. 
accommodations required to move from the initial mathematical model to actual predictions. ${ }^{57}$ Stirling's approximation is among the simpler of such ad hoc fixes.

This sort of thing leads some philosophers to a despair, to something along the lines from Duhem:

Our physical theories do not pride themselves on being explanations; our hypotheses are not assumptions about the very nature of material things. Our theories have as their sole aim the economical condensation and classification of experimental laws. (Duhem, 1906, p. 219) ${ }^{58}$ Agreement with experiment is the sole criterion of truth for a physical theory. (Duhem, 1906, p. 21)

Here the whole of theoretical science is regarded as a black box whose sole purpose and achievement is to catalog experimental outcomes. Just as the hope of a simple isomorphism between abstract model and worldly structure seems too optimistic, this fictionalist dismissal of any descriptive content seems too pessimistic, an overreaction to the complexities of scientific modeling. Though we may well be at a complete loss as to how quantum mechanics relates to the world, this is hardly true of the many other cases we have touched on; in kinetic theory, fluid dynamics, and practical chemistry, we have a fair idea of what our models are onto, where they are deficient, and why they work well nonetheless.

Still, one chastening conclusion does seem warranted: it appears unlikely that any general uniform account of how mathematics applies to the world could cover the wide variety of cases. To take just a few of the examples we have noted along the way, the point particle model of an ideal gas works effectively for dilute gases because the occupied volume is negligibly small compared to the total volume ${ }^{59}$ the 'continuum hypothesis' works effectively in fluid dynamics because there is a suitable 'plateau' between volumes too small to have stable temperature and volumes too large to have uniform temperature; the billiard ball model of a van der Waal gas works effectively because actual molecules do have fairly stable 'effective radii' ${ }^{60}$ Textbook examples could be multiplied:

57 See Wilson (2006, p. 26) for his 'lesson of applied mathematics': "Why do predicates behave so perversely? ... I believe the answer rests largely at the unwelcoming door of Mother Nature. The universe in which we have been deposited seems disinclined to render the practical description of the macroscopic bodies around us especially easy. ... Insofar as we are capable of achieving descriptive successes within a workable language ... we are frequently forced to rely upon unexpectedly roundabout strategies to achieve these objectives. It is as if the great house of science stands before us, but mathematics can't find the keys to its front door, so if we are to enter the edifice at all, we must scramble up backyard trellises, crawl through shuttered attic windows and stumble along half-lighted halls and stairwells." See also p. 452.

58 "The diverse principles or hypotheses of a theory are combined together according to rules of mathematical analysis. ... The magnitudes on which [the theorist's] calculations bear are not claimed to be physical realities, and the principles he employs in his deductions are not given as stating real relations among those entities; therefore it matters little whether the operations he performs do or do not correspond to real or conceivable physical transformations. All that one has the right to demand of him is that his syllogisms be valid and his calculations accurate.... Thus a true theory is not a theory which gives an explanation of physical appearances in conformity with reality; it is a theory which represents in a satisfactory manner a group of experimental laws. A false theory is not an attempt at explanation based on assumptions contrary to reality; it is a group of propositions which do not agree with experimental laws" (Duhem, 1906, pp. 20-21). See also Duhem (1906, p. 266).

59 For textbook treatment, see, e.g., Engel \& Reid (2006, p. 149).

60 For textbook discussion, see, e.g., McQuarrie \& Simon (1997, §16-7). 
We should discuss how good an approximation [a harmonic oscillator] is for a vibrating diatomic molecule.... Although the harmonic-oscillator potential may appear to be a terrible approximation to the experimental curve [of internuclear potential], note that it is, indeed, a good approximation in the region of the minimum. This region is the physically important region for many molecules at room temperature. ${ }^{61}$ The harmonic oscillator will be a good approximation for vibrations with small amplitudes. (McQuarrie \& Simon, 1997, pp. 163-164)

Treatments of applied mathematics also include careful analyses of when it is appropriate to replace a discrete variable with a continuous one:

The only difference between these last two equations is that the summation over discrete values of the variable ... has been replaced by integration over the range of the variable. The preceding comparison demonstrates that the continuous approximation is close to the exact result given by summation. In general, if the differences between the values the function can assume are small relative to the domain of interest, then treating a discrete variable as continuous is appropriate. This issue will become critical when the various energy levels of an atom or molecule are discussed. Specifically, the approximation ... will be used to treat translational and rotational states from a continuous perspective where direct summation is impractical. In the remainder of this text, situations in which the continuous approximation is not valid will be carefully noted. (Engel \& Reid, 2006, p. 290. See also p. 326)

Given the diversity of the considerations raised to delimit and defend these various mathematizations, anything other than a patient case-by-case approach would appear singularly unpromising.

Let us leave applied mathematics here and ask what we might learn from our historical survey about pure mathematics. One well-known metaphysical argument - that the role of mathematics in science confirms the objective existence of abstract mathematical entities $^{62}$ - must seem less compelling in light of what we have seen: if the scientist's actual claim is that some aspect of the world resembles a given abstract model in some respects, to some degree, within certain bounds, then it is hard to see why that abstract model must 'exist', whatever that comes to; all that matters for this sort of claim to have content is that the mathematical item be precisely characterized. (Think of describing the dynamics within an actual nuclear family by comparing it to another from novel or legend.) But more important for those of us looking to understand the actual practice of mathematics, there is a deep methodological moral to be drawn. Let me explain.

61 "Although the harmonic oscillator unrealistically allows the displacement to vary from 0 to $+\infty$, these large displacements produce potential energies so large that they do not often occur in practice."

62 I allude here to the Quine-Putnam indispensability arguments. See, e.g., Maddy (1997, pp. 103107) for exposition and references. Also Maddy (2007, pp. 314-317, 379-382). 
What we have traced is a more or less simultaneous rise of pure mathematics and reevaluation of applied mathematics. Before all these, back in Newton's or Euler's day, ${ }^{63}$ the methods of mathematics and the methods of science were one and the same; if the goal is to uncover the underlying structure of the world, if mathematics is simply the language of that underlying structure, then the needs of celestial mechanics (for Newton) or rational mechanics (for Euler) are the needs of mathematics. From this perspective, the correctness of a new mathematical method - say the infinitary methods of the calculus or the expanded notion of function ${ }^{64}$ - is established by its role in application. In contrast, after the developments we have been tracing, mathematics has been freed to pursue inquiries without application, it is encouraged to stock the warehouses with structures and leave the choices to the natural scientists, and even the mathematical constructs that do function in application do so with a new autonomy as freestanding abstract models. In this brave new world, where can the pure mathematician turn for guidance? How can we properly determine if a new sort of entity is acceptable or a new method of proof reliable? What constrains our methodological choices?

I think there are answers to these questions - practical mathematical answers not metaphysical ones - answers that appeal to the sort of means-ends considerations we touched on in the evolution of group theory - but that is a story for another day. ${ }^{65}$ For our purposes here, it seems to me that the only available moral is negative, that is, one apparent source of justificatory support that appeals to many observers is only that, is only apparent. What I have in mind is the sort of natural-science-based argument that is sometimes offered against the Axiom of Choice. ${ }^{66}$ Those familiar with the history ${ }^{67}$ will recall that Zermelo's introduction of the axiom in 1904 set off a fierce debate between its proponents and its opponents. This dispute had many strands - some metaphysical, some practical - but one feature of the axiom that particularly troubled many mathematicians was the so-called Banach-Tarski paradox: using the axiom, a sphere can be decomposed into finitely many parts and those parts reassembled into 2 spheres of the same size as the original. (The key is that the parts into which the sphere is cut are nonmeasurable, which is where Choice comes in.) This conclusion seems obviously absurd from a physical point of view, so Banach and

63 In fairness to Euler, I should note that Truesdell (1981, pp. 120-121) writes, "Today we look upon classical physics as providing us with mathematical models for the behavior of physical objects. We use these models with great caution, for we are deeply aware of their limitations ... The Bernoullis had no idea that they were dealing with models; like Galileo, they thought that nature herself spoke in mathematics. Euler in his middle life began to perceive how much the mathematician replaced nature by his own conceptions." I confess that I do not know what aspect of Euler's thought Truesdell has in mind here.

64 In his treatment of the wave equation, D'Alembert required the initial shape of a vibrating string to be given by an analytic expression (so as to be twice differentiable), but Euler insisted that functions should not be limited in this way, because the most common initial conditions - for example, a plucked string - do not satisfy this requirement. The result was a major revision of the notion of function, a firm push toward the modern set-theoretic conception. See Maddy (1997, pp. 116-123) for discussion and references.

65 See, e.g., Maddy (1997, §§III.4-III.6, and 2007, §IV.3).

66 In Maddy (1997, §§III.4-III.6), I argue that the mathematical goal of warehouse stocking coupled with set theory's aim of encompassing classical mathematics generates some very general methodological maxims for set theory - like 'include as many isomorphism types as you possibly can' - but here we are interested in the prospects for a more direct sort of empirical confirmation or disconfirmation.

67 See Moore (1982). 
Tarski's result is sometimes taken as evidence against Choice, as something akin to a false prediction. $^{68}$

If we have assimilated the lesson of the third strand of our story of how applied mathematics became pure, the strand that highlights the role of abstract models that resemble the world only partially and within certain limits, then the reply to this argument against Choice is straightforward: if physical regions are not literally modeled by sets of ordered triples of real numbers, then we cannot assume that all consequences of our mathematical theory of those sets will hold for those regions; therefore, we cannot conclusively draw our false empirical conclusion. What is perhaps slightly less obvious is that the full force of the third thread is not needed to defend Choice here; the second thread is enough, the one leading to the well-stocked warehouses of abstract structures.

To see this, ignore the third thread and suppose for the sake of argument that physical regions are literally modeled by subsets of $\mathbb{R}^{3}$. Then the argument goes like this: working in a set theory with the Axiom of Choice, we perform the Banach-Tarski construction, which is physically ridiculous; we conclude that Choice has been empirically disconfirmed. But isn't it at least as reasonable to conclude that the full power set of $\mathbb{R}^{3}$ was a poor choice as a model for physical regions? Indeed, given the many internal mathematical considerations in favor of the axiom, ${ }^{69}$ wouldn't it be considerably more reasonable to conclude that physical regions are more effectively modeled by measurable subsets of $\mathbb{R}^{3}$ ? If, for example, our set theory includes sufficient large cardinals, we might count Banach-Tarski as a good reason to model physical space in $L(\mathbb{R})$, where all sets of reals are measurable. ${ }^{70}$ In any case, the suggestion is that what is disconfirmed is the claim that regions are best modeled by $\wp\left(\mathbb{R}^{3}\right)$, not the Axiom of Choice. ${ }^{71}$ Once we have those wellstocked warehouses, any candidate for an empirical confirmation or disconfirmation of the mathematics is more reasonably viewed as confirming or disconfirming the claim that the right model has been identified. If this is right, then perhaps the added force of the third thread is more consequential for the practice of science - cautioning us against regarding all questions about our mathematical models as real physical issues - than it is for the practice of mathematics.

From this I think it is clear that considerations from applications are quite unlikely to prompt mathematicians to restrict the range of abstract structures they admit. It is just possible that as-yet-unimagined pressures from science will lead to profound expansions of the ontology of mathematics, as with Newton and Euler, but this seems considerably less likely than in the past, given that contemporary set theory is explicitly designed to be as inclusive as possible. ${ }^{72}$ More likely, pressures from applications will continue to influence

68 Fraenkel et al. (1973, p. 83) given references to this sort of reaction from Borel and others. They also note that Hausdorff had a similarly 'paradoxical' result in 1914, 10 years before BanachTarski.

69 See, e.g., Maddy (1997, pp. 54-57) for summary and references.

70 The existence of a supercompact cardinal implies that all sets of reals in $L(\mathbb{R})$ are Lebesgue measurable. See Jech (2003, pp. 650-653) or Kanamori (2003, §32). (Jech’s hypothesis is actually the existence of a superstrong cardinal, but the existence of a supercompact is considered the more natural hypothesis - a generalization of measurability - and it implies the existence of a superstrong.)

71 See Urquhart (1990, p. 152) for a similar conclusion in the context of Field's nominalization project.

72 For that matter, any hint of an important structure unrealized in the universe of sets would most likely motivate such an expansion whether the structure originated in science or not! 
which parts of the set-theoretic universe we attend to, as they did in the case of Dirac's delta function; ${ }^{73}$ in contemporary science, for example, the needs of quantum field theory and string theory have both led to the study of new provinces of the set-theoretic universe. ${ }^{74}$

There is much more to be said on all these complex topics, but let me stop here. I hope to have given some sense of how relations between mathematics and natural science have evolved over the centuries, more or less in tandem with the rise of pure mathematics. Seeing how these developments have peeled the mathematics off the world should lead us to a more accurate understanding both of how mathematics works in application and of the nature of pure mathematics. In particular, we should be alert to the ways that applied mathematics can generate phantom physical questions, and we should no longer expect science to provide the sort of methodological guidance for mathematics that it once did. This leaves us with the open question of how the methodological decisions of contemporary mathematics are properly justified, but that, again, is a story for another day.

Acknowledgments. My thanks to David Malament, John Rapalino, and Mark Wilson for their help. Distinct ancestors of this paper were presented as the Gauss Lecture in Dresden in October 2006 and the Ambrose/Tymoczko Lecture at Smith College in February 2007; I am grateful to the organizers and the audiences of both.

\section{BIBLIOGRAPHY}

Arfken, G. (1985). Mathematical Methods for Physicists (third edition). San Diego, CA: Academic Press.

Benacerraf, P. (1973). Mathematical truth. Reprinted in Benacerraf and Putnam [1983], pp. 403-420.

Benacerraf, P., \& Putnam, H., editors. (1983). Philosophy of Mathematics (second edition). Cambridge: Cambridge University Press.

Brown, T., LeMay, E., Bursten, B., \& Murphy, C. (2006). Chemistry: The Central Science (tenth edition). Upper Saddle River, NJ: Pearson-Prentice Hall.

Calinger, R. (1975). Euler's "Letters to a Princess of Germany" as an expression of his mature scientific outlook. Archive for History of Exact Sciences, 15, 211-233.

Carnap, R. (1932). The elimination of metaphysics through logical analysis of language. Reprinted in Ayer, A. J., editor, Logical Positivism. New York: Free Press [1959], pp. 60-81.

Carnap, R. (1950). Empiricism, ontology and semantics. Reprinted in Benacerraf and Putnam [1983], pp. 241-257.

73 The delta function takes the value 0 except at $x=0$, but its overall integral is 1 . This is impossible, of course, because the so-called function is 0 almost everywhere, which guarantees an integral of 0 . Dirac noted that it could nevertheless be used "for practically all purposes of quantum mechanics without getting incorrect results" (quoted by Pais in Pais et al., 1998, p. 7). (This function actually played a role as early as the 1890s in Heaviside's operational calculus. See van der Pol \& Bremmer (1959, pp. 1-5, 62-66) for the history.) Efforts to rigorize the delta function eventually led to Schwartz's theory of distributions or generalized functions (see van der Pol \& Bremmer, 1950, p. 74, or Zemanian, 1965).

74 See, e.g., Emch (1972) and Witten (1998). 
Cohen, B., \& Smith, G. (2002a). Introduction to Cohen and Smith [2002b]. In Cohen and Smith [2002b], pp. 1-32.

Cohen, B., \& Smith, G., editors. (2002b). Cambridge Companion to Newton. Cambridge: Cambridge University Press.

Duhem, P. (1906). The Aim and Structure of Physical Theory (Wiener, P., translator) Princeton, NJ: Princeton University Press, 1954.

Einstein, A. (1905). On the movement of small particles suspended in a stationary liquid demanded by the molecular-kinetic theory of heat. Reprinted in his Investigations of the Theory of Brownian Movement (Fürth, R., editor, Cowper, A. D., translator). Mineola, NY: Dover [1956], pp. 1-18.

Einstein, A. (1949). Autobiographical notes. In Schilpp, P. S., editor, Albert Einstein: Philosopher-Scientist. La Salle, IL: Open Court, pp. 1-105.

Emch, G. (1972). Algebraic Methods in Statistical Mechanics and Quantum Field Theory. New York: Wiley.

Emch, G., \& Liu, C. (2002) The Logic of Thermo-Statistical Physics. Berlin: Springer.

Engel, T., \& Reid, P. (2006). Thermodynamics, Statistical Thermodynamics, and Kinetics. San Francisco, CA: Pearson-Benjamin-Cummings.

Feynman, R., Leighton, R., \& Sands, M. (1964). The Feynman Lectures on Physics, vol. 2. Reading, MA: Addison-Wesley.

Fourier, J. (1822). The Analytical Theory of Heat (Freeman, A., translator). Mineola, NY: Dover, 1955.

Fraenkel, A., Bar-Hillel, Y., \& Levy, A. (1973). Foundations of Set Theory (second edition). Amsterdam: North Holland.

Galileo, G. (1638). Two New Sciences. (second edition, Drake, S., editor and translator). Toronto: Wall \& Emerson, 1974.

Gödel, K. (1964). What is Cantor's continuum problem? Reprinted in Benacerraf and Putnam [1983], pp. 470-485.

Guicciardini, N. (2002). Analysis and synthesis in Newton's mathematical work. In Cohen and Smith [2002b], pp. 308-328.

Hall, A. R. (2002). Newton versus Leibniz: From geometry to metaphysics. In Cohen and Smith [2002b], pp. 431-454.

Halliday, D., Resnick, R., \& Walker, J. (2005). Fundamentals of Physics. Hoboken, NJ: John Wiley \& Sons.

Ihde, A. (1964). The Development of Modern Chemistry. New York: Dover, 1984.

Jech, T. (2003). Set Theory (third millennium edition, revised and expanded). Berlin: Springer.

Kanamori, A. (2003). The Higher Infinite (second edition). Berlin: Springer.

Kline, M. (1972). Mathematical Thought from Ancient to Modern Times. New York: Oxford University Press.

Machamer, P. (1998a). Galileo's machines, his mathematics, and his experiments. In Machamer [1998b], pp. 53-79.

Machamer, P., editor (1998b). Cambridge Companion to Galileo. Cambridge: Cambridge University Press.

Maddy, P. (1997). Naturalism in Mathematics. Oxford: Oxford University Press.

Maddy, P. (2007). Second Philosophy. Oxford: Oxford University Press.

Malament, D. (1996). In defense of a dogma: why there cannot be a relativistic quantum mechanics of (localizable) particles. In Clifton, R., editor, Perspectives on Quantum Reality. Amsterdam: North Holland, pp. 1-10. 
McQuarrie, D., \& Simon, J. (1997). Physical Chemistry. Sausalito, CA: University Science Books.

Moore, G. H. (1982). Zermelo's Axiom of Choice. New York: Springer.

Newton, I. (1687). The Principia: Mathematical Principles of Natural Philosophy (Cohen,

B. and Whitman, A., translator). Berkeley, CA: University of California Press, 1999.

Nye, M. J. (1972). Molecular Reality. London: MacDonald.

Pais, A., Jacob, M., Olive, D., \& Atiyah, M. (1998). Paul Dirac: The Man and His Work (Goddard, P., editor). Cambridge: Cambridge University Press.

Partington, J. R. (1957). A Short History of Chemistry (third edition). New York: Dover, 1989.

Perrin, J. (1913). Atoms (Hammick, D., translator). Woodbridge, CT: Ox Bow Press, 1990.

Resnik, M. (1997). Mathematics as a Science of Patterns. Oxford: Oxford University Press.

Rice, J. (2007). Mathematical Statistics and Data Analysis (third edition). Belmont, CA: Thomson.

Shapiro, A. (2002). Newton's optics and atomism. In Cohen and Smith [2002b], pp. 227-255.

Silverman, A. (2003). Plato's Middle Period Metaphysics and Epistemology. In Zalta, E. N., editor, The Stanford Encyclopedia of Philosophy (Summer 2003 Edition). Available from: http://plato.stanford.edu/archives/sum2003/entries/plato-metaphysics/.

Slowik, E. (2005). Descartes's physics. In Zalta, E. N., editor, The Stanford Encyclopedia of Philosophy (Fall 2005 Edition). Available from: http://plato.stanford.edu/archives/ fall2005/entries/descartes-physics/.

Smith, C., \& Wise, N. (1989). Energy and Empire: A Biographical Study of Lord Kelvin. Cambridge: Cambridge University Press.

Smith, G. (2002). The methodology of the Principia. In Cohen and Smith [2002b], pp. 138-173.

Stein, H. (1990). On Locke, "the Great Huygenius, and the incomparable Mr. Newton". In Bricker, P. \& Hughes, R. I. G., editors, Philosophical Perspectives on Newtonian Science. Cambridge, MA: MIT Press, pp. 17-47.

Tritton, D. J. (1988). Physical Fluid Dynamics (second edition). Oxford: Oxford University Press.

Truesdell, C. (1960). The field viewpoint in classical physics. Reprinted in his [1984], pp. 21-40.

Truesdell, C. (1968). A program toward rediscovering the rational mechanics of the age of reason. In his Essays in the History of Mechanics. Berlin: Springer-Verlag, pp. 85-137.

Truesdell, C. (1981). The role of mathematics in science as exemplified by the work of the Bernoullis and Euler. Reprinted in his [1984], pp. 97-132.

Truesdell, C. (1984). An Idiot's Fugitive Essays on Science (second edition). New York: Springer-Verlag.

Uffink, J. (2007). Compendium of the foundations of classical statistical mechanics. In Butterfield, J. \& Earman, J., editors, Handbook of Philosophy of Physics. Amsterdam: North Holland, pp. 923-1073.

Urquhart, A. (1990). The logic of physical theory. In Irvine, A., editor, Physicalism in Mathematics. Dordrecht: Kluwer, pp. 145-154.

van der Pol, B. \& Bremmer, H. (1950). Operational Calculus. Cambridge: Cambridge University Press.

Wedberg, A. (1955). Plato's Philosophy of Mathematics. Westport, CT: Greenwood, 1977. Wilson, M. (2006). Wandering Significance. Oxford: Oxford University Press. 
Wise, N. (1981). The flow analogy to electricity and magnetism. Archive for History of Exact Sciences, 25, 19-70.

Witten, E. (1998). Magic, mystery, and matrix. Notices of the American Mathematical Society, 45, 1124-1129.

Zemanian, A. H. (1965). Distribution Theory and Transform Analysis. New York: Dover.

DEPARTMENT OF LOGIC AND PHILOSOPHY OF SCIENCE

UNIVERSITY OF CALIFORNIA AT IRVINE

IRVINE, CA 92697-5100, USA

E-mail: pjmaddy@uci.edu 